\title{
GH-MAP: translation system for sibling language pair Gujarati-Hindi
}

\author{
Kalyani A. Patel · Jyoti S. Pareek
}

Received: 27 April 2012 / Accepted: 24 December 2012/Published online: 12 March 2013

(C) CSI Publications 2013

\begin{abstract}
India is a linguistically rich country having eighteen constitutional languages, which are written in ten different scripts. Indian languages are highly inflectional with a rich morphology, relatively free word order, and default sentence structure as subject object verb. Many of them are structurally similar called sibling languages. Hindi and Gujarati languages are such siblings. The paper briefly describes GH-MAP; a rule based token mapping system developed by us for translation between sibling language pair Hindi and Gujarati. GH-MAP system performs effective word-for-word translation using simple and computationally inexpensive methods and minimal lexical resources. Issues of syntactic, semantic and structural divergence in translation using GH-MAP are resolved with the help of special empirical rules. The aim of GH-MAP is not to produce high quality translation in the sense of linguistics; rather it has been developed to produce correct working translation sufficient to cross the language barrier. The system was evaluated on the test bed obtained from FIRE 2010, literature on Gandhiji and ELRA-W0037. For establishing relevance of the model, 'into-Gujarati' BLEU, PER and METEOR score have been calculated.
\end{abstract}

K. A. Patel $(\square)$

K. S. School of Business Management, Gujarat University, Ahmedabad, India

e-mail: drkalyaniapatel@yahoo.com

J. S. Pareek

Department of Computer Science, Gujarat University,

Ahmedabad, India

e-mail: drjyotipareek@yahoo.com
Keywords GH-MAP - Rule based translation - Sibling language $\cdot$ Gujarati $\cdot$ Hindi

\section{Introduction}

Translation is defined as the task of transforming an existing text written in a source language, into an equivalent text in the target language. It is certainly possible that multiple perfect translations for a given source sentence may exist. These translations may vary in word choice or in word order even when they are using the same words. There are many approaches for machine translation such as rule-based, statistical-based and example-based. The statistical-based and example-based machine translation needs large size of bilingual corpus suitable for languages with indifferent structure. The rule-based machine translation needs linguistic knowledge of both the languages. As Gujarati and Hindi languages are sibling language, the grammatical differences and inexistence of some words are limited. Therefore, at lexical level some translation rules may be sufficient for one-to-one mapping of token. It can be made possible to resolve translation problem with iteration by adding new rules to the existing machine translation system. The paper describes our approach a rule based token mapping system for translation between language pair Gujarati and Hindi. One of the motivating aspects is that the efforts to develop applications like machine translation from English to Indian Languages, Indian Language to Indian Language have been taken up by different research groups in India [1]. It is observed that no work has been done for Gujarati. Another motivating aspect is that it is easier to create machine translation for a pair of related languages [2]. 


\section{Related work}

The efforts to develop applications like machine translation for closely related languages have been taken up by different research groups. Few of them are listed below:

- Apertium is an open source platform for developing machine translation. Armentano-Oller et al. [3] develops machine translation between Spanish and Catalan, Spanish and Galician using Apertium. Machine translation system of Apertium uses shallow transfer approach for translation. It comprise of following components to get translation for a given input sentence.

- Morphology analyzer.

- Part of speech tagger.

- Lexical transfer.

- Structural shallow transfer.

- Morphological generator.

- Post generator (e.g. Spanish de + el $=$ del, Catalan el + institute $=$ l'institut $)$

- RUSLAN is an unidirectional machine translation system for close languages Czech and Russian [4], it uses direct approach for translation. It would require no pre-editing but for high quality output it would require post editing. RUSLAN comprises of following processes to obtain translation for a given input sentence.

- Automatic pre-processing (performs national \& special characters conversion, coding and sentence boundaries recognition).

- Czech morphological analyser.

- Russian syntactico-semantic analyser.

- Morphological synthesis of Russian.

- CESILKO is a machine translation system for Czech and Slovak [2]. The system uses the method of direct word-for-word translation, justified by the similarity of syntactic constructions of both languages. CESILKO comprises of domain-related bilingual glossaries (incl. single- and multiword terminology), general bilingual dictionary and following processes to obtain translation for a given input sentence.

- Morphological analysis of Czech.

- Morphological disambiguation.

- Morphological synthesis of Slovak.

- Turkish and Crimean Tatar machine translation system [5] uses the method of disambiguated word-for-word translation. The grammars of the two languages are very similar, and each morpheme usually has a corresponding morpheme with or without change. The translation system comprises following processes to obtain translation for a given input sentence.

- Morphological analysis of Turkish text.

- Morphological disambiguation.

- Context dependent and grammatical translation rules.

- One-to-one translation of words.

- Morphological generation of Crimean Tatar text.

- The machine translation system between closely related languages Irish and Scottish [6]. Irish and Scottish are closely related languages of the Indo-European Family. Translation system comprises of following processes to obtain translation for a given input sentence.

- Irish standardization.

- POS tagging, stemming and chunking.

- Word sense disambiguation.

- Syntactic transfer.

- Lexical Transfer.

- Scottish post-processing.

\section{GH-MAP system}

Hindi and Gujarati are closely related languages. They reveal similarity both at lexical and grammatical level. As a result rule base for translation of their lexical category i.e. noun, verb, gender, number, tense etc., seems certainly possible. It is feasible to form rules whereby target language words can be derived regularly and productively from existing stem words via morphological processes and sub-string substitution. In addition, many Hindi words have the same meaning in Gujarati-especially the tatsam words (directly borrowed from Sanskrit) can be directly transliterated (e.g. Gujarati word અગ્ $\{\text { agni }\}^{1}$ is transliterate to Hindi word अग्नि $\{$ agni $\}[\text { fire }]^{2}$ ). Due to sibling properties, sentence from one language can be mapped to sentence in another language by substituting each word group in source language by appropriate word group in the target language,

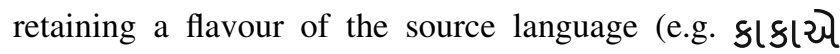
બેન્કમાંથી ગઈકાલે 1000 ર. ઉપાડયા. \{kAkAe benkmAMTI gikAle $1000 \mathrm{rU}$. upAdyA $\}$ is translated wordfor-word into Hindi as काका ने बेन्क में से कल 1000 रु. निकाले $\mathrm{I}\{\mathrm{kAkAe}$ benk meM se kl $1000 \mathrm{rU}$. nikAle $\})$. Taking advantage of the above mentioned features of sibling languages, GH-MAP a rule based token mapping

\footnotetext{
1 The \{\} bracket contains WX notation equivalence for Hindi or Gujarati character.

2 The [] bracket contains English language word corresponding to Hindi/Gujarati language word.
} 
system for translation between language pair Gujarati and Hindi has been designed and implemented. To implement token mapping translation system, GH-MAP requires tables for monolingual words, bilingual words and rules to bootstrap dictionary for token mapping between Hindi and Gujarati words. The rule based token mapping uses simple and computationally inexpensive methods such as:

- For One-to-One Token mapping [7].

- Sub-string substitution

Hindi and Gujarati language have many words with small difference in sub string. To take advantage of this particular feature of language pair, rule based token mapping develop substring substitution rule to derive target word using substring substitution. Hindi and Gujarati languages have many words with little difference of substrings. For example, the Gujarati word 'વસ્તी' \{vswI\}[population] can be derived by substituting substring ' $ब$ ' $\{b\}$ by' $a$ ' $\{v\}$ in Hindi word 'बस्ती' $\{$ bswI $\}$. A table has been designed for the substring substitution rule to take maximum advantage of feature of the languages. The rule table stores Hindi substrings corresponding to Gujarati substrings, location of substitution and flag. The value in the flag manages application of the rule at every occurrence of substring in a source string or in a specific context to derive target word. The initial substring substitution rule base was derived from illustration given by Nayak ${ }^{3}$ and analyzing word forms in both the languages. The rule base was then tested with specific documents and was updated iteratively by analyzing the words which did not get translated or were incorrectly translated.

- Generating and mapping inflected noun/verb/adjectiveladverb forms

A morpheme is defined as the smallest part of a language that can be regularly assigned a meaning. Both Gujarati and Hindi languages depend heavily on suffixes for morphological changes. There are number of suffixes for achieving declension of verbs, nouns, adjectives and adverbs. Further, in both the languages there are situations when morphological changes in the adjectives and adverbs are also required depending upon the number and gender of the corresponding noun, pronoun or verb. Since the number of suffixes is limited, we feel that instead of purely word-based operations if we focus on

\footnotetext{
${ }^{3}$ Nayak [12]
}

the suffixes, then in many situations significant amount of lookup efforts would be saved. As Hindi and Gujarati are inflectional languages, in every document inflected word (noun, verb, adjective, and adverb) appears. Such words can be reduced to a common shorter form called a stem, by the successive removal of the longest possible suffix. Mapping of stem and their suffixes has been used to generate inflected word and their appropriate translation for better performance e.g. the different forms of inflected words would improve the coverage; obtained for languages. For example, with Gujarati stem word clof \{bAlYk $\}$, Gujarati suffixes $\{0\}$ and $\{\mathrm{I}\}$ can be used, similarly with Hindi stem word बच्च $\{$ bcc $\}$, Hindi suffixes $\{$ e $\}$ and $\{I\}$ can be used. Thus, different forms of inflected words are obtained, such as: બાળફો, બાળકી, बच्चे, बच्ची [baby boys, baby girl]. Similarly, adjective such as 'बडा' \{barA\} [big] and 'छोटा' \{Cota [small], ends with 'r? in the masculine singular, ' $\Rightarrow$ ' in masculine plural and in the feminine singular and feminine plural. Such words can be translated using stem suffix rules as stated.

The lists of suffix governing inflections are simple and few in number [8]. To translate inflected words, a table of stems and suffix rules has been designed. The table of stem word stores Hindi and Gujarati stem words, and there are separate tables for Hindi suffix and Gujarati suffix.

\section{- Dictionary word mapping}

Bilingual dictionary mapping is used by GH-MAP system for domain specific words which have different typologies, phrases which include compound words and idioms, invariable (uninflected) karaks, pronouns, adjectives and adverbs.

- In every document certain domain specific words, are neither inflected nor derived and are typologically different such as, अचार $\{\mathrm{acAr}\}$ [pickle] in Hindi can be અથાણું \{aWANuM $\}$ in Gujarati. Such words are stored in a language specific table and their relations are stored into word map table for better performance.

- In every document case endings (Karak) are important in both the languages. These karakas [9] are syntactico-semantic in nature and thus help in capturing a certain degree of semantic. A karaka is defined as a participant in an action. Because Hindi and Gujarati are close, karaka can be mapped quite effectively such as, ने $\{$ ne $\}$ in 
Hindi would be 引 $\{\mathrm{ae}\}$ in Gujarati. To translate karaks (case markers, post position markers) bilingual tables (Hindi and Gujarati words in the same table) have been designed, because of their high frequency occurrence, less variety and wide distribution in the documents.

- In every document adjectives, pronouns, adverbs, auxiliary verb and conjunction are important terms in both the languages. Some of the non-inflected (invariable) forms of adjectives, pronouns, auxiliary verb, conjunction and adverbs are directly mapped for better performance such as, आप $\{\mathrm{aAp}\}[\mathrm{you}]$ in Hindi would be તમ \{wme $\}$ in Gujarati. To translate pronouns, adjectives and adverbs, bilingual tables (Hindi and Gujarati words in the same table) have been designed, because of their high frequency occurrence, less variety and wide distribution in the documents.

- Phrases and compound words are the words formed from two or more words. In every document, language specific phrases are used such as, नाक-नक़शा \{nAk-nkSA\} [look] in Hindi would be દેખl વ \{xeKAv\}in Gujarati. To translate multi-word expression including compound words and idioms, a bilingual phrase table has been designed.

- Though token mapping engine works quite well for the most of the sentences, it is noted that some useful conditions or properties of the grammatical elements play a vital role in the sentential environment of both the languages. Differences in properties (gender/number/person) require many-to-many or one-to-many token mapping in certain cases of post position markers (e.g. का $\{\mathrm{kA}\} /$ की $\{\mathrm{kI}\} /$ के $\{\mathrm{ke}\}$ [of] would map to નો \{no $\} / \sigma\{\{n I\} / \sigma ु$ \{nuM $\})$ pronouns (e.g. उसका \{uskA\} [his/her/their] would map to તેનો \{weno\}/તની \{wenI\}/તેનું \{wenu\}), adjectives (e.g. नया \{nayA $\}[$ new] would map to નવું \{nvuM\}/નવો \{nvA\}/नવी \{nvI\}) and adverbs (e.g. धीमा \{XImA\}[slow] would map to ધીમો \{XImo\}/ધીમું \{XImu\}).

- For One-to-Many or Many-to-Many or Manyto-One Token mapping

\section{- Empirical rules [10]}

For one-to-many and many-to-many token mapping an exclusive empirical rule layer have been designed on the token mapping layer in GH-MAP system. The empirical rules resolve syntactic and semantic divergence issues and improve translation quality of GH-MAP system. Such as in Hindi sentence if 'पुरानी' \{purAnI $\}$ refers to a noun 'इमारत' \{imArw $\}$ having feminine gender which is translated to a neuter gender noun 'મકાન' \{mkAn\} in Gujarati, then 'पुरानी' is translated into ' भूनुं' \{jUnuM\} (e.g. (H) वह इमारत पुरानी है. $\{v h$ imArw purAnI $\mathrm{hE}$ \} is translated into $(\mathrm{G})$ તે મકાન જૂનું छ. \{we mkAn jUnuM Ce\}). The default sentence structure of Hindi language and Gujarati language is subject object verb (SOV).Owing to such a structural similarity between Hindi and Gujarati languages, syntactic rule that governs the transfer of the source structure to target structure are not required.

\subsection{Translation mechanism}

As shown in Fig. 1, the GH-MAP system takes a sentence in source language and search for phrases in the source sentence. If the sentence in source language contains phrase, it would be mapped with equivalent target language phrase. Then tokenize the sentence. Translate token using empirical rules which depend on grammatical properties of predecessor tokens. If the source token does not get translated using empirical rule, then translate token using token mapping engine as shown in Fig. 2. Then, remove translation disambiguation using empirical rules which depend on grammatical properties of successor tokens.

Token Mapping Engine performs word-for-word mapping to translate source language token into target language token. As shown in Fig. 2, the token mapping engine search for each token in table of karaka, pronouns, adjectives, adverbs and in the table of domain specific words to get equivalent target language token. If match is not found then it would identify stem of a source language token and search stem and suffix in the table of stem-suffix rule to get equivalent target language inflected word. Even after the search, word is not found then it would search substrings in source token. If match is found then the next step would be to derive target language token by substring substitution and transliteration of remaining string of the token. Else it would transliterate the source token into target token, considering token as a proper name of Hindi language or the word of foreign language (English and Persian) or 'tatsam' (borrowed words from Sanskrit).

\subsection{User interface}

Interface design for GH-MAP system is depicted in Fig. 3. Through this interface, a user can select the source 


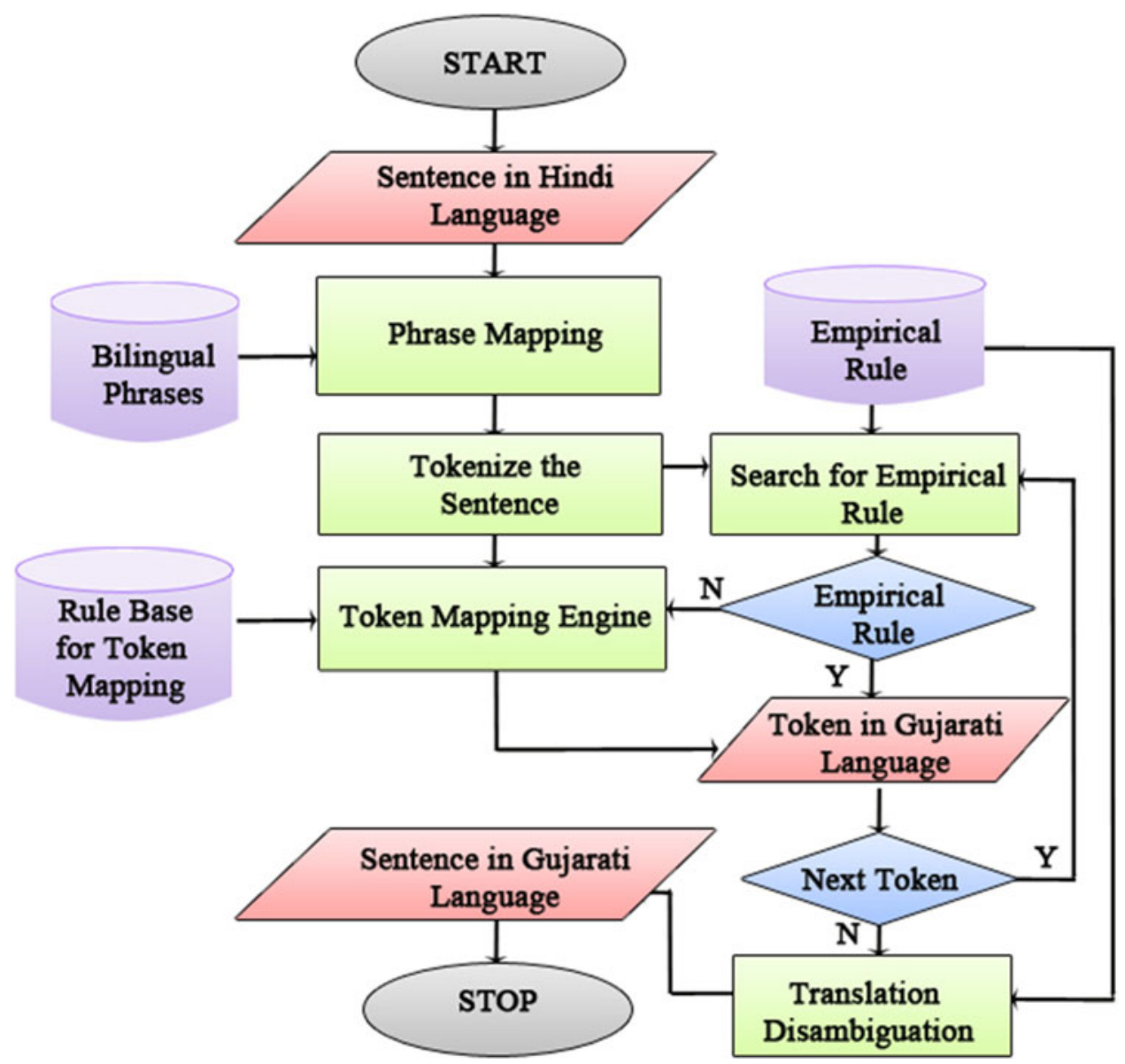

Fig. 1 GH-MAP process flow

language, the mode of input can be manual or user can get it from text file or database table. The 'Interpret' button is used to start translation as explained in process flow in Fig. 1. The 'save' option helps the user to save output in temporary table for further analysis. The 'Add New' button refresh the interface to accept new input and the 'Resource Table' button is to assist the admin to add new rules to rule base. The Fig. 3 shows the stage when the user selects Hindi as a source language with mode of input as manual. The input textbox enables and accepts input from the user in source language. After pressing Interpret button the output in target language will be displayed in the output textbox.

\subsection{Contribution of various resources in GH-MAP}

The resources used in GH-MAP system are as follows:

- Sub string substitution rules to derive target language word.

- Hindi and Gujarati stem words (i.e. semantically minimal, morpheme style units) and suffix mapping rules.
- Tables of bilingual dictionary for domain specific words, synonyms, cases (karaks), pronouns, adjectives, adverbs and phrases. ${ }^{4}$

- Empirical Rules

For the estimation of percentage contribution of various resources in translation, the document set consisting of documents from literature on Gandhiji, ${ }^{5}$ FIRE $2010^{6}$ and ELRA-W0037 $7^{7}$ are used. Total 1000 Hindi sentences (15,550 words) were translated by GH-MAP to Gujarati for evaluation purpose. The Table 1 shows the contribution of various resources used in GH-MAP for translation. The percentile contribution of various resources in translation is calculated as follows.

\footnotetext{
${ }^{4}$ Phrase here is defined as a string of adjacent words and not as a syntactic constituent.

5 Gandhiji [13], [14].

${ }^{6}$ Forum for Information Retrieval Evaluation (FIRE, 2010).

7 ELRA : Evaluation and Language Resources Distribution Agency, France, The EMILLE/CIIL corpus from www.elda.org.
} 


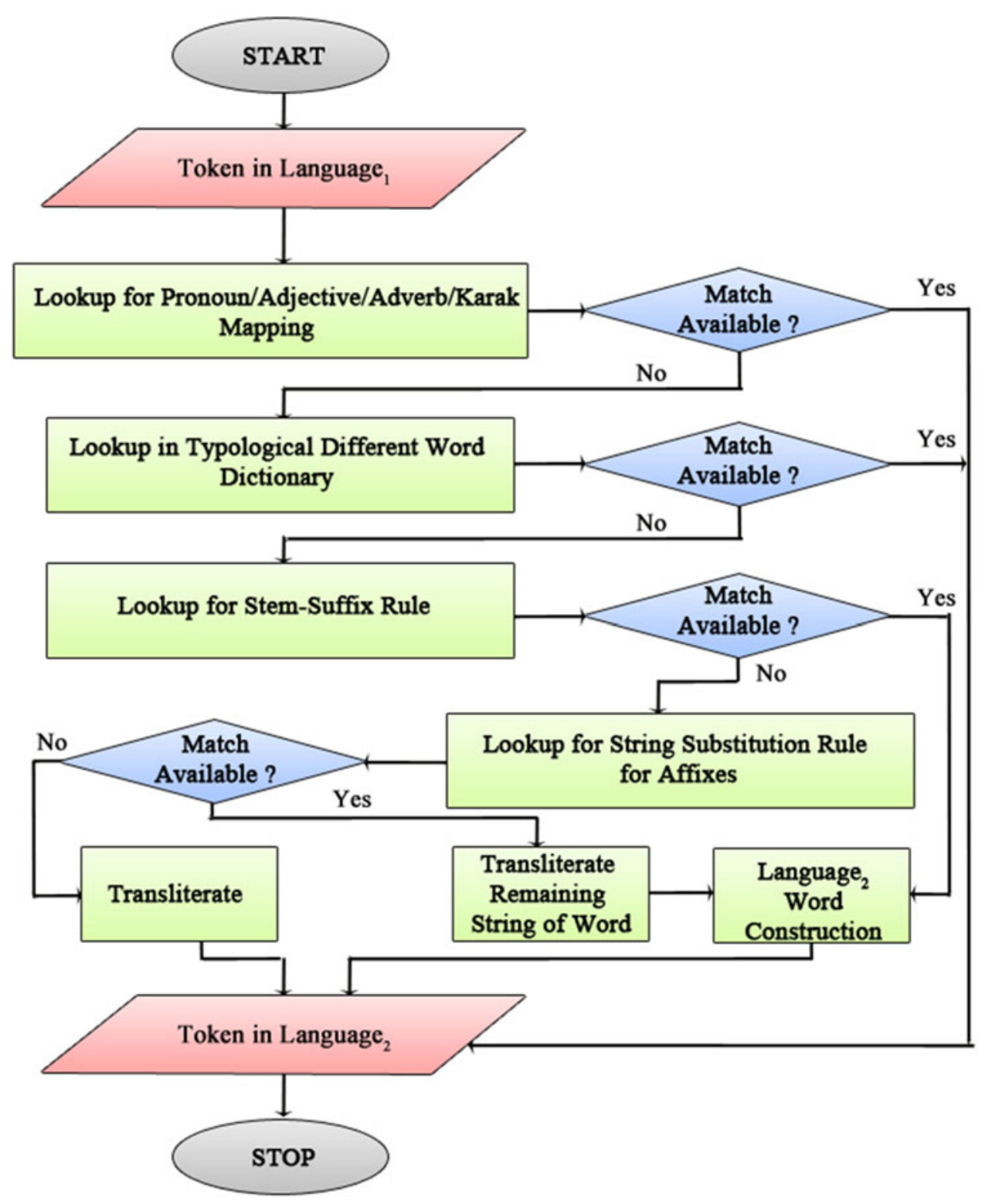

Fig. 2 Token mapping engine

Contribution in translation $=100 *$ (Number of words translated using particular resources)/(Total number of words translated)

GH-MAP has facilitated simple interface to add new rules. Total contribution of rule base approach in translation can be seen from the Table 1 and Fig. 4. This justifies appropriateness of rule base approach for the proposed model.

\section{Evaluation of GH-MAP system}

Evaluating a machine translation system using machine evaluation is much faster, easier and cheaper compared to human evaluations. A common element of machine evaluation is the usage of a set of test sentences for which we already have human translations, called reference translations. However, the usage of a single reference may be considered as the evaluation towards a particular translation style. In order to mitigate against this and to reflect the diversity of the best possible good translations, we should use multiple references. We have used two reference translations to evaluate candidate translation (output from GH-MAP system).

- Reference ${ }_{1}$ translation (We have asked the language expert to translate the documents word-for-word (i.e. word-based), retaining the flavour of source language. Such translation is feasible because Gujarati and Hindi are structurally similar languages.) 


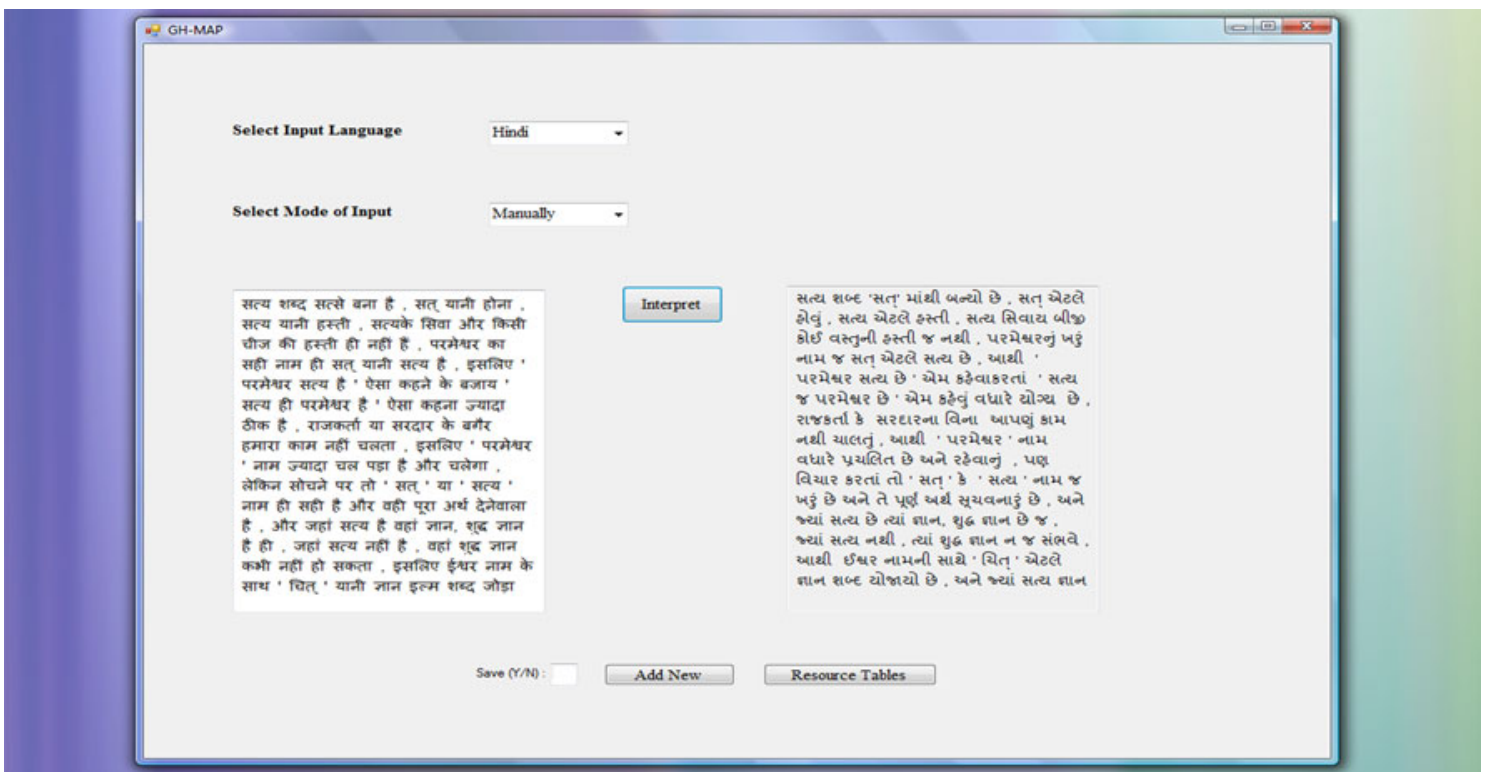

Fig. 3 User interface for GH-MAP

- Reference 2 translation (Language model based translation is obtained from a bilingual corpus. Translation for each test sentence is extracted from bilingual corpus FIRE 2010, literature on Gandhiji and ELRA-W0037.)

Machine evaluation, software has been developed. The software accepts file1 (the target language document generated by GH-MAP, i.e. candidate translation) and file2 (reference translation) as an input file and gives translation quality in terms of three scores, viz. PER, BLEU and METEOR. It is observed that BLEU, METEOR and PER score is designed to approximate human judgment at a corpus level. Considering 'into-Gujarati' (word-order, superficial differences in the character sequences of the tokens, exact query term (keyword)) characteristics in mind, we have performed following steps on sentence from 'candidate translation' and sentence from 'reference translation' before calculating BLEU, PER and METEOR score.

- Substitute synonyms

- Remove suffixes to generate stem

- Normalize token

- Remove stop words

- Perform the word reordering

For Example,

Input Sentence: आडवाणी के विरुद्ध बाबरी मसजिद विध्वंस मामला दायर, बाबरी मसजिद विधवंस मामले में आडवाणी के विरुद्ध कानूनी कार्यवाही की शुरुआत I \{AdvANI ke viruxX bAbrI msjix viXvMs mAmlA $x A y r$, bAbrI msjix viXvMs mAmle meM AdvANI ke viruxX kAnUnI kAryvAhI kI SuruAw\}.

\subsection{Candidate translation}

Output Sentence: અSવાણીના વિરુધ્ધ બાબરી મા્જિદ વિદ્વાસ કેસ દાખલ, બાબરી મા્જિદ વિદ્વાસ કેસમાં અડવાણીના विરુद્ધ કાનૂની કાર્યवाફીની શરુત \{AdvANInA viruxX bAbrI msjix viXvMs kes xAKl, bAbrI msjix viXvMs kesmAM AdvANInA viruxX kAnUnI kAry$v A h I n I$ SruAw .

Table 1 Contribution of various resources in translation

\begin{tabular}{|c|c|c|}
\hline \multirow[t]{2}{*}{$\begin{array}{l}\text { Resources used in the GH-MAP } \\
\text { for translation of words }\end{array}$} & \multicolumn{2}{|c|}{$\begin{array}{l}\text { Resources used by GH-MAP for } \\
\text { translation }\end{array}$} \\
\hline & $\begin{array}{l}\text { No of words } \\
\text { translated using } \\
\text { the resource }\end{array}$ & $\begin{array}{l}\text { Contribution in } \\
\text { translation }(\%)\end{array}$ \\
\hline $\begin{array}{l}\text { Table for substring substitution } \\
\text { rules }\end{array}$ & 467 & 3 \\
\hline Table for stem-suffix rules & 3,577 & 23 \\
\hline $\begin{array}{l}\text { Table for mapping domain } \\
\text { specific words }\end{array}$ & 623 & 4 \\
\hline $\begin{array}{l}\text { Table for mapping phrase } \\
\text { tokens }\end{array}$ & 310 & 2 \\
\hline Table for mapping case rules & 1,868 & 12 \\
\hline Table for mapping pronoun & 158 & 1 \\
\hline $\begin{array}{l}\text { Table for mapping adjectives \& } \\
\text { adverbs }\end{array}$ & 158 & 1 \\
\hline Empirical rules & 623 & 4 \\
\hline $\begin{array}{l}\text { Tables for mapping auxiliary } \\
\text { verbs, conjunction and others }\end{array}$ & 2,030 & 13 \\
\hline Transliteration & 5,736 & 37 \\
\hline Total & 15,550 & 100 \\
\hline
\end{tabular}


Fig. 4 Influence of various approaches in translation

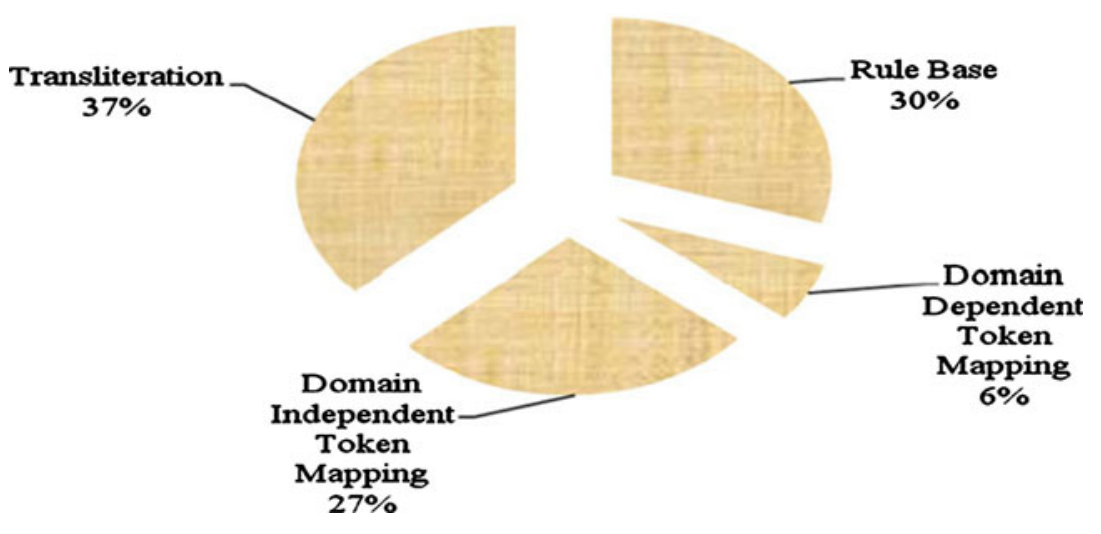

4.2 Sentence from reference 2

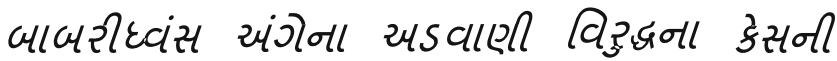
કાર્યવાડી શર થઇ, બાબરીધ્વસસમાં પોતાની સંડીવણી માટે અડવાણી વિરુ્ધ કાયદેસર રીતે કાર્યવાહીનો પ્રારંભ. \{bAbrIXvMs aMge AdvANI viruxXnA kesnI kAryvAhI Sru Wi, bAbrIXvMsmAM powAnI sMdovNI mAte AdvANI viruxX kAyxesr kAryvAhIno prArMB\}.

Thus, after performing the earlier discussed steps on sentence from candidate and sentence from reference 2 translation, we will obtain refined sentence of reference ${ }_{2}$ and candidate as explained below.

- Substitute synonym:

This operation replaces synonym in reference ${ }_{2}$ sentence. For example 'પ્રંંભ' $\{$ prArMB\} will be replaced by 'શરુઆત' \{SruAw\} and 'કાયદેસર' \{kAyxesr\} will be replaced by કાનૂની\{kAnUnI\}.

- Remove suffixes to generate stem.

This operation removes suffixes 'ની' $\{n I\}$, 'નl' $\{n A\}$ from candidate sentence and 'નો ' $\{n o\}$, 'માં' $\{m A M\}$ from reference ${ }_{2}$ sentence, the resulting refined sentence will have 'કાર્યવવાફી' \{kAryvAhI\}, 'કેસ'\{kes\} and 'આS वlણી' $\{a d v A N I\}$ respectively.

- Normalize token:

This operation replace 'બાબરીદ્વંસ' $\{b A b r I X v M s\}$ token of reference 2 sentence with 'બાબરી મસ્જિદ વિધ્વંસ' $\{b A b r I$ msjix viXvMs\} tokens of candidate sentence for token normalization.

- Remove stop words:

This operation removes the stop words (e.g. common, meaningless words, which are not indexed) like માટે
\{mAte\}, પોતાની \{powAnI\}, રીતે \{rIwe\} from reference $_{2}$ sentence as well as from candidate sentence.

- Perform the word reordering:

Finally, the task of reordering the tokens of the reference $_{2}$ sentence with respect to candidate sentence would be performed to get refined sentence. The resulting refined sentence will be:

Refined Sentence of Candidate Translation: અડ વાણી વિરુદ્ધબાબરી મસ્જિદ વિધ્વંસ કેસ દાખલ બાબરી મસ્જિદ વિદ્વંસ કેસ અડવાણી વિરુદ્ધ કાનૂની કાર્યવાહી શરુઆત [AdvANInA viruxX bAbrI msjix viXvMs kes xAKl bAbrI msjix viXvMs kes AdvANI viruxX kAnUnI kAryvAhI SruAw\}.

Refined Sentence of Reference 2 Translation: અડ વાણી વિરુદ્ધબાબરી મસ્જિદ વિધ્વંસ કેસ દાખલ બાબરી મસ્જિદ વિદ્વંસ કેસ અડવાણી વિરુદ્ધ કાનૂની કાર્યવાહી શરુઆત/AdvANInA viruxX bAbrI msjix viXvMs kes xAKl bAbrI msjix viXvMs kes AdvANI viruxX kAnUnI kAryvAhI SruAw\}.

Evaluation of GH-MAP system, tests its suitability and effectiveness to overcome language barrier for cross lingual information extraction and retrieval. Table 2 shows quality exploration after performing above mentioned steps

Table 2 Corpus level BLEU, METEOR and PER score

Hindi to Gujarati translation

\begin{tabular}{llll}
\hline & BLEU & PER & METEOR \\
\hline $\begin{array}{l}\text { Candidate \& reference } \\
\text { considering 'into Gujarati' [only } \\
\text { substitution of synonym and token } \\
\text { normalization] }\end{array}$ & 0.88 & 0.09 & 0.96 \\
$\begin{array}{l}\text { Candidate \& reference } \\
\text { considering 'into Gujarati' }\end{array}$ & 0.78 & 0.19 & 0.91 \\
\hline
\end{tabular}


on 1,000 sentences of candidate translation and reference ${ }_{1}$ and reference ${ }_{2}$ translation. The GH-MAP is primarily developed for query translation task of cross lingual information extraction and retrieval, but looking at its effectiveness it can be used for machine translation where literary translation are not required (e.g. news, manual of gadgets), localization, development of GurjerNet-Gujarati WordNet and development of multilingual dictionary [11].

\section{Conclusion}

In this paper, we have discussed advent of GH-MAP system for query translation. GH-MAP is an effective rule based token mapping system for translation between sibling language pair Gujarati and Hindi. As Gujarati and Hindi are structurally similar languages, GH-MAP generates target language sentence retaining a flavour of the source language. It should be noted that translation is not performed here in the sense of linguistics, but word-forword translation is performed. It requires limited linguistic effort and tools for achieving the said goal. Result of BLEU, METEOR and PER score, demonstrates the potential advantage and accuracy of our approach. Though further improvement can be made, the test results obtained are motivating and encouraging.

\section{Appendix 1}

See Appendix Table 3.

\section{References}

1. Agrawal S (2008) Development of resources and techniques for processing of some Indian languages. Invited lecture-at linguistic Data Consortium, Upenn

2. Hajic J, Hric J, Kubon V (2000) Machine translation of very close languages. In: Proceedings of the 6th Applied Natural Language Processing Conference, pp 7-12

3. Armentano-Oller C, Carrasco RC, Corb'1- Bellot AM, Forcada ML, Ginest'1-Rosell M, Ortiz-Rojas S, P'erez-Ortiz JA, Ram'ırez$S^{\prime}$ anchez G, S' anchez-Mart' inez F, Scalco MA (2006) Open-source Portuguese-Spanish machine translation. In: Computational Processing of the Portuguese Language, in Proceedings of the 7th International Workshop on Computational Processing of Written and Spoken Portuguese, PROPOR. Lecture Notes in Computer Science, vol 3960. Springer, pp 50-59

4. Hajic J (1987) Ruslan: an MT system between closely related languages. In: Proceedings of the 3rd Conference of The European Chapter of the Association for Computational Linguistics, Copenhagen, Denmark, pp 113-117

5. Altintas K, Cicekli I (2002) A machine translation system between a pair of closely related languages. In: Proceeding of International Symposium on Computer and Information Sciences

6. Scannell KP (2006) Machine translation for closely related language pairs. In: Proceedings of Language Resource Evaluation Conference

7. Patel K, Pareek J (2009) GH-MAP-rule based token mapping for translation between sibling language pair: Gujarati-Hindi. In: Proceedings of International Conference on Natural Language Processing

8. Ramanathan A, Bhattacharyya P, Hegde J, Shah R, Sasikumar M (2008) Simple syntactic and morphological processing can help English-Hindi statistical machine translation. In Proceedings of International Joint Conference on NLP

9. Bharti A, Vineet C, Sangal R (1994) Natural language processing: a paninian perspective. Prentice-Hall of India, New Delhi

10. Patel K, Pareek J (2010) Rule base to resolve translation problems due to differences in gender properties in sibling language pair Gujarati-Hindi. In: Proceedings of IEEE International Conference on Computer and Communication Technology

Table 3 WX-Notation

\begin{tabular}{|c|c|c|c|c|c|c|c|c|c|c|c|c|c|c|c|}
\hline $\mathrm{a}$ & $\mathrm{A}$ & $\mathrm{i}$ & I & $\mathrm{u}$ & $\mathrm{U}$ & $\mathrm{Q}$ & $\mathrm{Q}$ & $\mathrm{k}$ & $\mathrm{K}$ & $\mathrm{g}$ & $\mathrm{G}$ & $\mathrm{f}$ & $\mathrm{c}$ & $\mathrm{C}$ & $\mathrm{j}$ \\
\hline अ & आ & इ & ई & 3 & ऊ & ॠ & ऋ & क् & ख् & ग् & घ् & ङ् & च् & छ् & ज् \\
\hline W & $x$ & $X$ & $\mathrm{n}$ & $\mathrm{p}$ & $\mathrm{P}$ & B & B & $\mathrm{L}$ & $\mathrm{e}$ & $\mathrm{E}$ & 0 & $\mathrm{O}$ & $\mathrm{aM}$ & $\mathrm{aH}$ & $\mathrm{az}$ \\
\hline थ् & द् & ध् & न् & प् & फ् & ब् & भ् & लृ & ए & ऐ & ओ & औ & अं & अः & अ \\
\hline $\mathrm{J}$ & $\mathrm{F}$ & $\mathrm{t}$ & $\mathrm{T}$ & $\mathrm{d}$ & $\mathrm{D}$ & $\mathrm{N}$ & w & $\mathrm{M}$ & $\mathrm{y}$ & $\mathrm{r}$ & 1 & $1 \mathrm{Y}$ & $\mathrm{S}$ & $\mathrm{R}$ & $\mathrm{s}$ \\
\hline झ् & ज् & ट् & ర् & ड् & ढ् & ण् & त् & म् & य् & र् & ल् & চ् & श् & ष् & स् \\
\hline $\mathrm{V}$ & $\mathrm{h}$ & & \multirow{2}{*}{\multicolumn{13}{|c|}{ राम $=$ र्+आ+म्+अ $(\mathrm{rAma}) \quad$ कृष्ण $=$ क्+ ऋ+ष्+ण्+अ(kqRNa) }} \\
\hline व् & ह् & & & & & & & & & & & & & & \\
\hline
\end{tabular}


11. Mohanty RK, Bhattacharyya P, Kalele S, Pandey P, Sharma A, Kopra M (2008) Synset based multilingual dictionary: insights, applications and challenges. In: Proceedings of the 4th Global WordNet Conference

12. Nayak R (2005) Nanha Kosh, 24th edn. (Bhag 1-2, HindiGujarati and Gujarati-Hindi). Anada Book Depo, Ahmedabad, pp 15-17
13. Gandhiji MK (1999) Maaraa Svapna nu Bhaarat. Navjivan Prakashan Mandir, Ahmedabad

14. Gandhiji MK (2007) Mangal prabhaat. Navjivan Prakashan Mandir, Ahmedabad 